\title{
Resultados de la cirugía de reparación valvular mitral en el Hospital Guillermo Grant Benavente de Concepción (2009-2020)
}

\author{
Enrique Seguel ${ }^{1,2}$, Héctor Rubilar ${ }^{3}$, Aldo Vera-Calzaretta ${ }^{4}$, Aleck Stockins $^{1,2}$, Roberto González ${ }^{1,2}$, Sebastián Ramirez $^{3}$. \\ 1. Universidad de Concepción, Facultad de Medicina, Departamento de Cirugía. \\ 2. Hospital Guillermo Grant Benavente de Concepción, Centro Cardiovascular. \\ 3. Universidad de Concepción, Alumno de Medicina. \\ 4. Universidad de Atacama, Facultad de Ciencias de la Salud, Departamento de Kinesiología.
}

Objetivo: Describir las características clínicas, resultados operatorios inmediatos y a 5 años de la cirugía de reparación valvular mitral.

Material y métodos: Estudio retrospectivo de la cohorte de pacientes operados de reparación valvular por insuficiencia mitral (IM) en el Hospital Guillermo Grant Benavente desde el 2009 hasta marzo 2020 $(\mathrm{N}=206)$. Se comparan los pacientes con IM primaria y secundaria en sus características clínicas, ecocardiográficas, técnicas quirúrgicas empleadas, cirugías asociadas, morbimortalidad operatoria, sobrevida y reintervenciones hasta el 30 de mayo de 2020.

Resultados: 124(60,2\%) hombres. Edad media $62,6 \pm 10,5$ años. La IM fue primaria en 134 y secundaria en 72 . En comparación con los pacientes portadores de IM primaria, aquellos con IM secundaria tuvieron más enfermedad coronaria $(69,4 \%$ versus $11,9 \%$; $\mathrm{p}<0,001)$ y mayor riesgo operatorio (EuroSCORE lo-

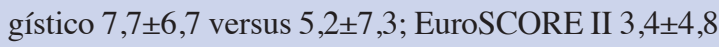

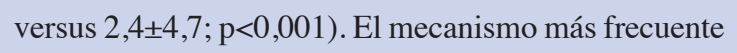
de IM primaria fue tipo II $(65,7 \%)$ y en las secundarias fue el tipo III $(48,6 \%)$ seguido del tipo I $(30,6 \%)$. Las IM primarias se corrigieron principalmente con procedimientos para disminuir el prolapso $(76,1 \%)$. En las secundarias la técnica más utilizada fue el implante de un anillo exclusivo (76,4\%). Hubo 116 cirugías asociadas y $10(4,9 \%)$ conversiones a reemplazo valvular. Hubo 57 complicaciones operatorias y fallecieron 12 $(5,8 \%)$ pacientes, $5(3,7 \%)$ con IM primaria y $7(9,7 \%)$ con IM secundaria. La sobrevivencia global a 5 años fue $83,5 \%$ (90\% en las primarias y $78 \%$ en las secundarias) y hubo 6 reintervenciones.

Conclusiones: La cirugía de reparación valvular, tanto en pacientes con IM primaria como secundaria, tuvo una baja mortalidad operatoria y excelentes resultados a 5 años.

Palabras clave: Cirugía cardiaca; válvula mitral; insuficiencia mitral: reparación valvular. 


\section{Results of Surgical Mitral Valve Repair at Guillermo Grant Benavente Hospital, Concepción, Chile}

Aim: To describe the clinical characteristics, operative and long term results of surgical mitral valve repair.

Material and methods: Retrospective study of the cohort of patients undergoing valve repair due to mitral regurgitation (MR) at the Guillermo Grant Benavente Hospital from 2009 to March 2020 (N = 206). Patients with primary and secondary MR were compared on clinical and echocardiographic characteristics, surgical techniques, associated surgeries, operative morbidity and mortality, survival and reinterventions up to May 30, 2020.

Results: 124 (60.2\%) were men. The average age was $62.6 \pm 10.5$ years. Type of MR was primary in 134 and secondary in 72. Compared to patients with primary MR, those with secondary MR had more coronary artery disease $(69.4 \%$ versus $11.9 \%$; $\mathrm{p}<0.001)$ and greater operative risk (logistic EuroSCORE $7.7 \pm 6,7$ versus 5.2 \pm 7.3 ; EuroSCORE II $3.4 \pm 4.8$ ver- sus $2,4 \pm 4.7 ; p<0.001)$. The most frequent mechanism of MR was type II in primary (65.7\%) and type III (48.6\%) followed by type I (30.6\%) in secondary MR. Primary MR was corrected mainly with procedures to decrease prolapse (76.1\%). In secondary MR the main technique used was the implantation of an exclusive ring (76.4\%). There were 116 associated surgeries and $10(4.9 \%)$ conversions to valve replacement. There were 57 operative complications and 12(5.8\%) patients died, 5 (3.7\%) with primary MR and 7 (9.7\%) with secondary MR. Overall survival at 5 years was $83.5 \%$ (90\% in primary MR and $78 \%$ in secondary MR) and there were 3 reoperations.

Conclusions: Valve repair surgery in both primary and secondary MR patients was associated to a low operative mortality and excellent results at 5 year post surgery.

Keywords: Surgery, cardiac; mitral valve; mitral insufficiency, mitral valve repair. 


\section{Introducción:}

La prevalencia de enfermedad valvular en la población general de países desarrollados está estimada en un 2,5\%, siendo la insuficiencia mitral la valvulopatía más frecuente. El prolapso valvular por enfermedad degenerativa puede encontrarse hasta en el 2,4\% de la población.Sin embargo, sólo un 3,5\% de ellos tiene una insuficiencia mitral (IM) significativa. ${ }^{1}$ A estos pacientes se suman aquellos con enfermedad coronaria, quienes pueden tener una IM significativa hasta en el $12 \%$ de los casos y aquellos con disfunción del ventrículo izquierdo, que pueden presentar una IM significativa hasta en el $20 \%$ de los casos. ${ }^{1}$

En los países en vías de desarrollo la situación epidemiológica es diferente y existe un predominio de enfermedad reumática, la que puede representar hasta un $72 \%$ de las causas de enfermedad valvular. ${ }^{1}$

La cirugía de reparación mitral fue descrita por varios autores desde principios de los años 60, quienes utilizaron jaretas, el cierre de las comisuras y otras técnicas para corregir la IM. ${ }^{2}$ Sin embargo fue Alain Carpentier, en Francia, quien sistematizó el análisis de la válvula, los mecanismos causales de la insuficiencia y propuso los principios técnicos para corregirlos, lo que permitió extender la reparación mitral a todo el mundo. ${ }^{3,4}$

La reparación de la válvula es considerara actualmente como el tratamiento de elección de la IM y según los registros de la Sociedad de Cirujanos Torácicos de Estados Unidos representa más del $60 \%$ de las cirugías sobre esta válvula. 5,6

En nuestro país el primer reporte de reparación valvular mitral fue publicado por el Dr. Juan Dubernet, del grupo de la Universidad Católica, en la Revista Chilena de Cirugía el año 1977.7 Unos años más tarde, en 1994, el Dr. Ricardo Zalaquett reportó una serie de 16 pacientes intervenidos desde diciembre de 1991 con excelentes resultados a 11 meses dando inicio a una larga experiencia con la técnica. ${ }^{8}$

A pesar de esta tendencia a nivel nacional y mundial, la mayoría de los casos de patología mitral intervenidos hasta mediados de esta década en nuestro hospital aún lo son por causa reumática y la cirugía mitral consistió principalmente en el reemplazo valvular.

Los primeros pacientes con enfermedad mitral degenerativa susceptible de reparar fueron diagnosticados recién el año 2009. En esa época, tanto las técnicas diagnósticas por ecocardiografía como las de reparación quirúrgica estaban consolidadas, lo que nos permitió iniciar la cirugía de reparación valvular mitral en nuestro hospital en ese año.
El objetivo de esta comunicación es describir nuestra experiencia en los primeros 11 años de reparación valvular mitral.

\section{Métodos:}

Se efectuó un estudio descriptivo de la cohorte de pacientes intervenidos de una reparación valvular mitral exclusiva o asociada a otras cirugías en el Hospital Guillermo Grant Benavente entre el año 2009 y abril del 2020 (N=206).

Los datos fueron extraídos del registro de cirugías cardiacas del centro cardiovascular y la base de datos del hospital y fueron anonimizados.

\section{Variables}

Se estudian las características demográficas, clínicas y el riesgo operatorio calculado por EuroSCORE (aditivo, logístico y II).

Se analizan los mecanismos de la IM según la clasificación de Carpentier. ${ }^{9}$

Se consideraron primarias a las insuficiencias causadas por lesión orgánica de la válvula y secundarias a aquellas causadas por mecanismo ventricular con indemnidad de la válvula (isquémica o por dilatación ventricular).

Se describen los tipos de reparación realizada, las cirugías asociadas, las conversiones a reemplazo por una prótesis, las complicaciones y la mortalidad operatoria.

\section{Seguimiento:}

El seguimiento se completó hasta el 31 de mayo de 2020. Los datos de sobrevida y causas de muerte se obtuvieron del Servicio de Registro Civil e Identificación.

Se estudiaron la mortalidad alejada y la necesidad de reintervenciones sobre la válvula.

\section{Análisis estadístico}

Se realizó un análisis descriptivo de las distribuciones porcentuales de variables nominales u ordinales. Se utilizaron medidas de tendencia central y de dispersión para las variables cuantitativas. Se utilizó el método Kaplan Meier para el estudio de la sobrervivencia, global y según etiología. Para el procesamiento estadístico de los datos se utilizaron los softwares estadísticos IBM SPSS 21.0 y Stata 14.

\section{Resultados:}

Entre enero de 2009 y abril de 2020 se realizaron 875 cirugías mitrales exclusivas o asociadas a otra intervención. De ellas, 206 correspondieron a reparaciones, lo que representa el 23,5\% de las cirugías. Esta proporción 


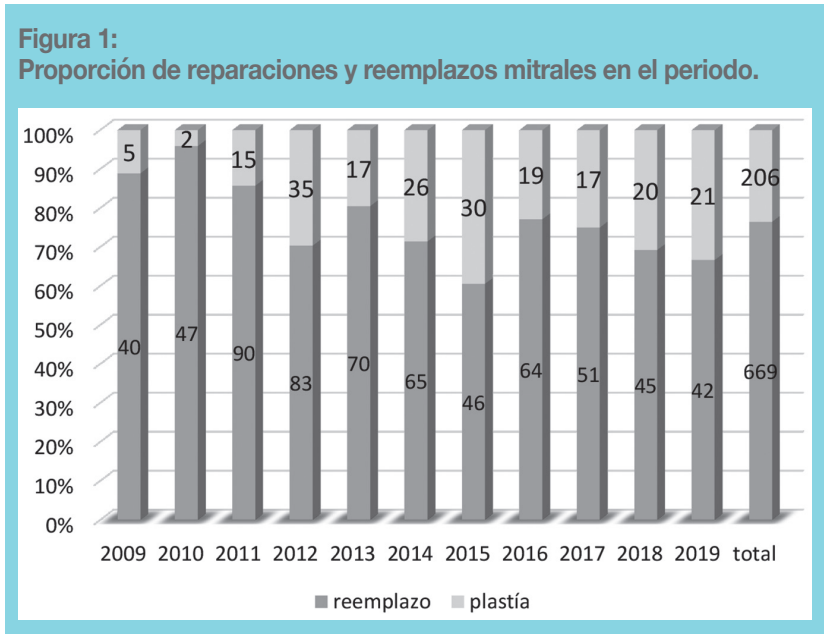

ha ido evolucionando con los años desde un $11 \%$ el 2009 hasta un 33,3\% el 2019. La Figura 1 muestra la proporción de reparaciones respecto del total de cirugías mitra- les en el periodo.

\section{Pacientes}

Corresponden a 124 hombres $(60,2 \%)$. La edad promedio

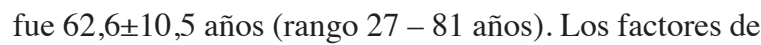
riesgo, patologías asociadas, capacidad funcional y riesgo operatorio se muestran en la Tabla 1.

En comparación con la IM primaria, los pacientes con IM secundaria presentaron más enfermedad coronaria $(69,4 \%$ versus $11,9 \% ; \mathrm{p}<0,001)$ y un mayor riesgo operatorio tanto por EuroSCORE aditivo $(7,4 \pm 4,6$ versus $4,9 \pm 4,4 ; p<0,001)$, como por EuroSCORE logístico $(7,7 \pm 6,7$ versus $5,2 \pm 7,3 ; \mathrm{p}<0,001)$ y EuroSCORE II $(3,4 \pm 4,8$ versus $2,4 \pm 4,7 ; \mathrm{p}<0,001)$.

\section{Ecocardiografía}

Los hallazgos del ecocardiograma pre operatorio se describen en la Tabla 2.

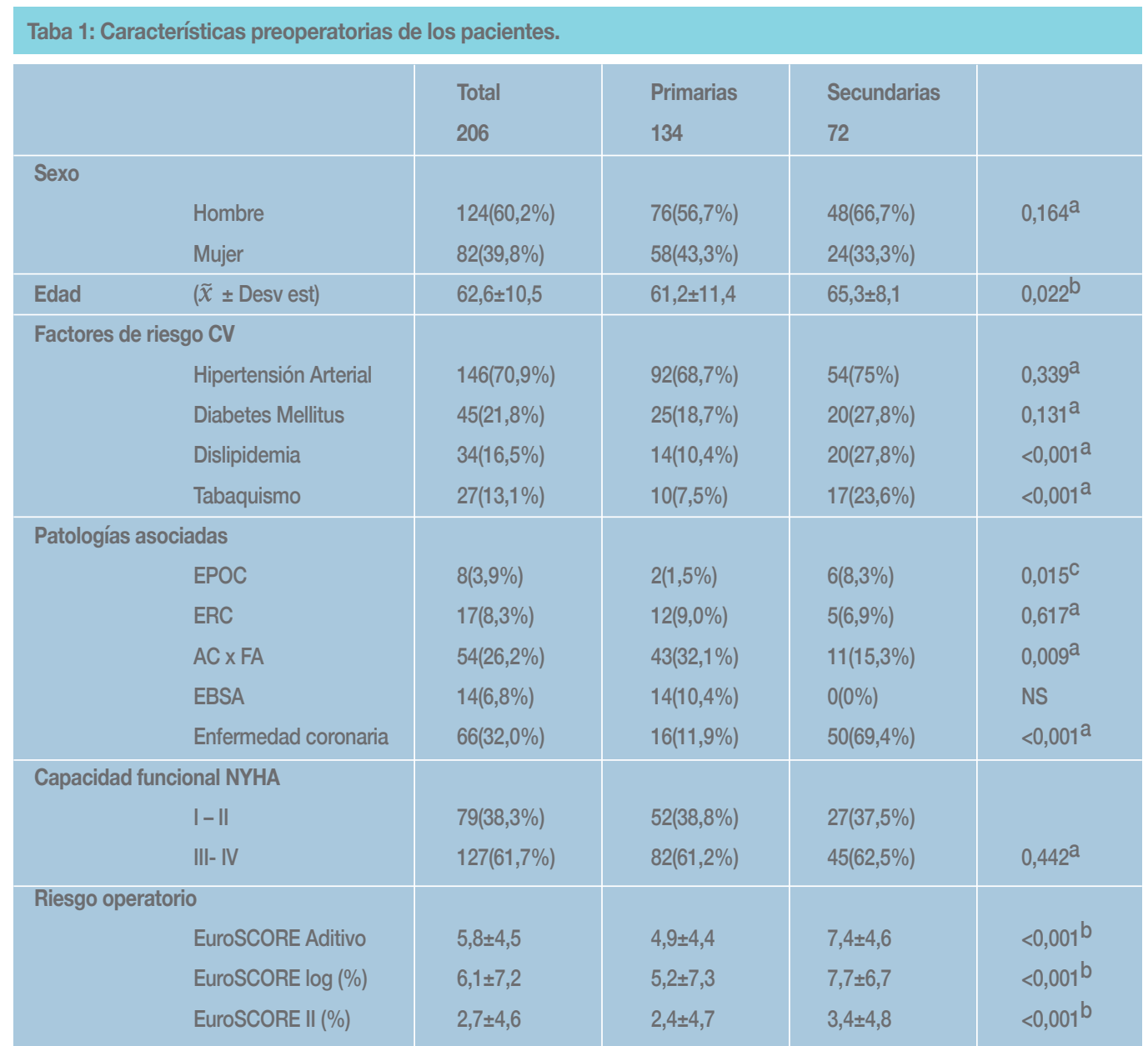

${ }^{a}$ Chi-cuadrado; ${ }^{b}$ Prueba $U$ de Mann Whitney; ${ }^{c}$ Prueba exacta de Fisher

EPOC: Enfermedad pulmonar obstructiva crónica. ERC: Enfermedad renal crónica. EBSA: Endocarditis bacteriana activa. AC $x$ FA: Fibrilación auricular 


\begin{tabular}{|c|c|c|c|c|}
\hline Severidad de la IM & $\begin{array}{l}\text { Total } \\
206\end{array}$ & $\begin{array}{l}\text { Primarias } \\
134\end{array}$ & $\begin{array}{l}\text { Secundarias } \\
72\end{array}$ & \\
\hline Moderada & $47(22,8 \%)$ & $18(13,4 \%)$ & $29(40,3 \%)$ & $<0,001^{a}$ \\
\hline Severa & $159(77,2 \%)$ & $116(86,6 \%)$ & $43(59,7 \%)$ & \\
\hline \multicolumn{5}{|l|}{ Valvulopatia asociada } \\
\hline Aortica & $33(16,0 \%)$ & $10(7,5 \%)$ & $23(31,9 \%)$ & $<0,001^{b}$ \\
\hline Tricúspide & $31(15,0 \%)$ & $23(17,2 \%)$ & $8(11,1 \%)$ & $0,247^{\mathrm{a}}$ \\
\hline \multicolumn{5}{|l|}{ Valores ecográficos } \\
\hline DSV (mm) & $42,3 \pm 9,9$ & $39,7 \pm 9,1$ & $47,9 \pm 9,3$ & $<0,001^{C}$ \\
\hline $\mathrm{DDV}(\mathrm{mm})$ & $60,7 \pm 9,0$ & $59,1 \pm 7,9$ & $64,3 \pm 10,1$ & $0,004^{C}$ \\
\hline FE $(\%)$ & $53,8 \pm 14,0$ & $57,8 \pm 12,5$ & $45,7 \pm 13,5$ & $<0,001^{C}$ \\
\hline $\mathrm{Al}(\mathrm{mm})$ & $51,9 \pm 8,3$ & $52,3 \pm 8,8$ & $50,9 \pm 7,3$ & $0,135^{\mathrm{C}}$ \\
\hline \multicolumn{5}{|l|}{ Mecanismo principal } \\
\hline Dilatación anillo & $45(21,8 \%)$ & $23(17,2 \%)$ & $22(30,6 \%)$ & $0,027^{a}$ \\
\hline Perforación velo & $8(3,9 \%)$ & $8(6,0 \%)$ & $0(0 \%)$ & NS \\
\hline Prolapso anterior & $35(17,0 \%)$ & $28(20,9 \%)$ & $7(9,7 \%)$ & $0,042^{a}$ \\
\hline Prolapso posterior & $57(27,7 \%)$ & $47(35,1 \%)$ & $10(13,9 \%)$ & $<0,001^{a}$ \\
\hline Prolapso bivalvular & $4(1,9 \%)$ & $4(3,0 \%)$ & $0(0 \%)$ & NS \\
\hline Prolapso comisural & $10(4,9 \%)$ & $9(6,7 \%)$ & $1(1,4 \%)$ & $0,090^{\mathrm{b}}$ \\
\hline Tracción & $31(15,0 \%)$ & $3(2,2 \%)$ & $28(38,9 \%)$ & $<0,001 a$ \\
\hline Retracción & $19(9,2 \%)$ & $12(8,9 \%)$ & $7(9,7 \%)$ & $0,856^{\mathrm{a}}$ \\
\hline
\end{tabular}

El mecanismo más frecuente en las IM primarias fue el tipo II, por prolapso de un segmento de la válvula, en un $65,7 \%$. Correspondieron a prolapso de velo posterior en 47 , velo anterior en 28 , comisulal en 9 y bi-valva en 4 pacientes. En las IM secundarias el mecanismo más frecuente fue el tipo III, por tracción o retracción del aparato sub valvular $(48,6 \%)$, seguido de tipo I, por dilatación anular $(30,6 \%)$. Los pacientes con IM secundaria tuvieron más enfermedad valvular aórtica asociada $(31,9 \%$ versus $7,5 \%$; $<<0,001)$.

\section{Cirugías}

Todas las cirugías se realizaron por esternotomía completa y con apoyo de ecocardiografía transesofágica (ETE) realizada por el anestesiólogo. En los casos de duda concurrió un cardiólogo a evaluar la válvula. Las técnicas de las reparaciones variaron en ambos grupos: en las IM primarias se hicieron principalmente procedimientos para disminuir el prolapso de los segmentos comprometidos $(76,1 \%)$. Se realizaron 43 resecciones de velo posterior, 4 transpocsiciones de cuerdas P2 a A2; 19 implantes de neocuerdas de PTFE y 36 cierres comisurales. En las secundarias la técnica más utilizada consistió en el implante exclusivo de un anillo, ajustado al tamaño de la válvula o sub dimensionado $(76,4 \%)$.

Todas las reparaciones incluyeron el implante de un anillo protésico. Se utilizaron diferentes anillos en diferentes periodos de acuerdo a la disponibilidad del hospital: Tailor® (St Jude Medical, flexible) entre los años 2009 - 2011 (N=18); Saddle® (St Jude Medical, rígido) del 2011 al 2013 (N=58); Annuloflo ${ }^{\circledR}$ (Carbomedics) del 2013 al 2016 (N=62); Physio® (Edwards) entre el 2016 y 2019 (N=60) y Physio II $($ Edwards) desde el 2019 en adelante $(\mathrm{N}=8)$.

Hubo 116 cirugías asociadas (65 cirugías coronarias, 31 reemplazos valvulares aórticos, 15 plastias tricuspídeas y 5 reemplazos de aorta ascendente), las que fueron más frecuentes en los pacientes con IM secundaria.

El resultado de la reparación se consideró satisfactorio en 196 pacientes, con una IM residual grado I o menor. En los otros 10 pacientes $(4,9 \%)$ hubo una IM residual grado II o mayor, sin poder lograr una reparación satisfactoria 


\begin{tabular}{|c|c|c|c|c|}
\hline & Total & Primarias & Secundarias & \\
\hline Tipo de reparación mitral & 206 & 134 & 72 & \\
\hline Anillo exclusivo & $71(34,5 \%)$ & $31(23,1 \%)$ & $40(55,6 \%)$ & $<0,001^{a}$ \\
\hline Anillo sub dimensionado & $21(10,2 \%)$ & $6(4,5 \%)$ & $15(20,8 \%)$ & $<0,001^{a}$ \\
\hline Parche de pericardio & $6(2,9 \%)$ & $6(4,5 \%)$ & $0(0 \%)$ & NS \\
\hline Resección de velo & $46(22,3 \%)$ & $43(32,1 \%)$ & $3(4,2 \%)$ & $<0,001^{a}$ \\
\hline Transposición de cuerdas & $5(2,4 \%)$ & $4(3,0 \%)$ & $1(1,4 \%)$ & $0,478^{b}$ \\
\hline Neo cuerda & $25(12,1 \%)$ & $19(14,2 \%)$ & $6(8,3 \%)$ & $0,221^{a}$ \\
\hline Cierre de comisura & $42(20,4 \%)$ & $36(26,9 \%)$ & $6(8,3 \%)$ & $0,002^{\mathrm{a}}$ \\
\hline Cierre de cleft & $11(5,3 \%)$ & $11(8,2 \%)$ & $0(0 \%)$ & NS \\
\hline Resección de vegetaciones & $3(1,5 \%)$ & $3(2,2 \%)$ & $0(0 \%)$ & NS \\
\hline Comisurotomìa & $6(2,9 \%)$ & $4(3,0 \%)$ & $2(2,8 \%)$ & $0,933^{b}$ \\
\hline Reimplante de musculo papilar & $1(0,5 \%)$ & $1(0,7 \%)$ & $0(0 \%)$ & NS \\
\hline \multicolumn{5}{|l|}{ Tipo de Anillo } \\
\hline St. Jude Medical Tailor & $18(8,7 \%)$ & $14(10,4 \%)$ & $4(5,5 \%)$ & \\
\hline St. Jude Medical Saddle & $58(28,2 \%)$ & $26(19,4 \%)$ & $36(50 \%)$ & \\
\hline Carbomedics Annuloflo & $62(30,1 \%)$ & $41(30,6 \%)$ & $21(29,2 \%)$ & \\
\hline Physio - Ring & $60(29,1 \%)$ & $47(35,1 \%)$ & $13(18,0 \%)$ & \\
\hline Physio- Ring II & $8(3,9 \%)$ & $6(4,4 \%)$ & $2(2,8 \%)$ & \\
\hline Tamaño ( $\tilde{x} \pm$ Desvest) & $29,7 \pm 2,3$ & $30,0 \pm 2,2$ & $29,1 \pm 2,3$ & \\
\hline Plastia frusta & $10(4,9 \%)$ & $6(4,5 \%)$ & $4(5,6 \%)$ & $0,731^{b}$ \\
\hline Tiempo de CEC ( $\tilde{x} \pm$ Desvest) & $84,8 \pm 36,0$ & $77,0 \pm 34,1$ & $102,7 \pm 34,4$ & \\
\hline \multicolumn{5}{|l|}{ Cirugías Asociadas } \\
\hline Coronaria & $65(31,6 \%)$ & $15(11,2 \%)$ & $50(69,4 \%)$ & $<0,001^{a}$ \\
\hline Aorta ascendente & $5(2,4 \%)$ & $1(0,7 \%)$ & $4(5,6 \%)$ & $0,032^{b}$ \\
\hline Valvular Aórtica & $31(15,0 \%)$ & $10(7,5 \%)$ & $21(29,2 \%)$ & $<0,001^{a}$ \\
\hline Valvular Tricúspide & $15(7,3 \%)$ & $10(7,5 \%)$ & $5(6,9 \%)$ & $0,891^{a}$ \\
\hline
\end{tabular}

${ }^{a}$ Chi-cuadrado; ${ }^{b}$ Prueba exacta de Fisher; ${ }^{c}$ Prueba U de Mann Whitney.

y fueron convertidas a reemplazo valvular con prótesis.

El detalle de las cirugías realizadas se muestra en la Tabla 3.

\section{Complicaciones y mortalidad}

Hubo 57 complicaciones operatorias, que incluyen 8 reintervenciones por sangrado.

No hubo diferencias en las complicaciones entre los grupos. Fallecieron $12(5,8 \%)$ pacientes, 5(3,7\%) en el grupo de IM primaria y $7(9,7 \%)$ en el grupo de IM secundaria, sin diferencia significativa. La principal causa de muerte fue shock refractario en 7 pacientes. Otros 3 pacientes fallecieron de muerte súbita, uno por taponamiento cardiaco y uno por falla orgánica múltiple.

El detalle de las complicaciones y mortalidad operatoria se presenta en la Tabla 4.

\section{Seguimiento}

El Seguimiento promedio fue de 5,3 años, durante los

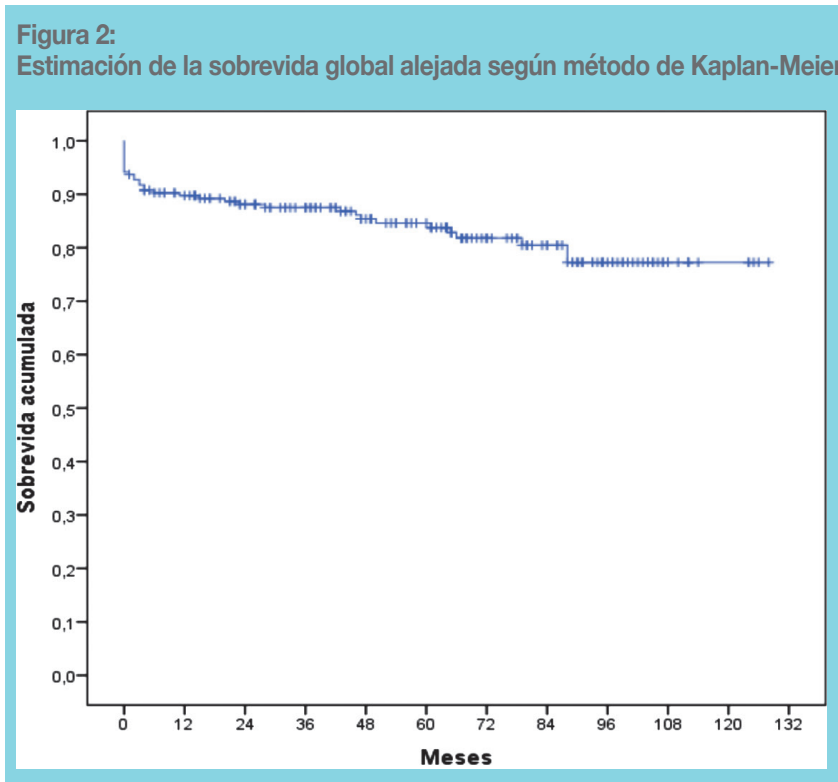




\begin{tabular}{|c|c|c|c|c|}
\hline & $\begin{array}{l}\text { Total } \\
206\end{array}$ & $\begin{array}{l}\text { Primarias } \\
134\end{array}$ & $\begin{array}{l}\text { Secundarias } \\
72\end{array}$ & \\
\hline Complicaciones & $57(27,7 \%)$ & $36(26,9 \%)$ & $21(29,2 \%)$ & $0,725^{\mathrm{a}}$ \\
\hline Cardiacas & $29(14,1 \%)$ & $20(14,9 \%)$ & $9(12,5 \%)$ & $0,633^{a}$ \\
\hline Arritmias & $27(13,1 \%)$ & $20(14,9 \%)$ & $7(9,7 \%)$ & $0,291^{a}$ \\
\hline Falla bomba & $4(1,9 \%)$ & $0(0 \%)$ & $4(5,6 \%)$ & NS \\
\hline Renales & $12(5,8 \%)$ & $7(5,2 \%)$ & $5(6,9 \%)$ & $0,615^{b}$ \\
\hline IRA & $11(5,3 \%)$ & $6(4,5 \%)$ & $5(6,9 \%)$ & $0,453^{b}$ \\
\hline ITU & $1(0,5 \%)$ & $1(0,7 \%)$ & $0(0 \%)$ & NS \\
\hline Respiratoria & $14(6,8 \%)$ & $7(5,2 \%)$ & $7(9,7 \%)$ & $0,221^{b}$ \\
\hline Neumonía & $7(3,4 \%)$ & $4(3,0 \%)$ & $3(4,2 \%)$ & $0,655^{b}$ \\
\hline VM $>24$ horas & $10(4,9 \%)$ & $3(2,2 \%)$ & $7(9,7 \%)$ & $0,017^{b}$ \\
\hline Neurológicas & $7(3,4 \%)$ & $3(2,2 \%)$ & $4(5,6 \%)$ & $0,210^{b}$ \\
\hline AVE & $2(1,0 \%)$ & $2(1,5 \%)$ & $0(0 \%)$ & NS \\
\hline Síndrome confusional & $1(0,5 \%)$ & $0(0 \%)$ & $1(1,4 \%)$ & NS \\
\hline Síndrome convulsivo & $4(1,9 \%)$ & $1(0,7 \%)$ & $3(4,2 \%)$ & $0,090^{b}$ \\
\hline Quirúrgicas & $16(7,8 \%)$ & $7(5,2 \%)$ & $9(12,5 \%)$ & $0,063^{a}$ \\
\hline Re op. por sangrado & $8(3,9 \%)$ & $3(2,2 \%)$ & $5(6,9 \%)$ & $0,096^{b}$ \\
\hline Mediastinitis & $4(1,9 \%)$ & $1(0,7 \%)$ & $3(4,2 \%)$ & $0,090^{b}$ \\
\hline Dehiscencia esternal & $1(0,5 \%)$ & $1(0,7 \%)$ & $0(0 \%)$ & NS \\
\hline Infección herida operatoria & $4(1,9 \%)$ & $3(2,2 \%)$ & $1(1,4 \%)$ & $0,673^{b}$ \\
\hline Mortalidad Operatoria & $12(5,8 \%)$ & $5(3,7 \%)$ & $7(9,7 \%)$ & NS \\
\hline
\end{tabular}

${ }^{a}$ Chi-cuadrado; ${ }^{b}$ Prueba exacta de Fisher.

que fallecieron 22 pacientes. La sobrevida global de la serie fue de $83,5 \%$ (Figura 2).

Las causas de muerte fueron: insuficiencia cardiaca en 6; muerte súbita (paro cardio respiratorio) en 5 ; sepsis en 4; neumonía en 3; trombo embolismo pulmonar en 2; síndrome urémico y hematoma retro peritoneal en 1 . La sobrevida a 5 años estimada por método de Kaplan Meier $90 \%$ para IM primaria y $78 \%$ para IM secundaria $(\mathrm{p}=0,01)$. (Figura 3 )

Hubo 6 reintervenciones sobre la válvula mitral: 5 por recurrencia de IM y 1 por endocarditis. Además se reoperó un paciente por un por leak perivalvular de prótesis aórtica y se instaló un marcapasos a 8 pacientes.

\section{Discusión:}

Si bien las técnicas e indicaciones de reparación mitral fueron descritas hace muchos años, fue recién en el año 2009 que comenzamos a intervenir pacientes con IM no
Figura 3:

Estimación de la sobrevida a 5 años por método de Kaplan - Meier según si la IM era primaria (verde) o secundaria (azul): $90 \%$ versus $78 \%$ respectivamente $(p=0,01)$.

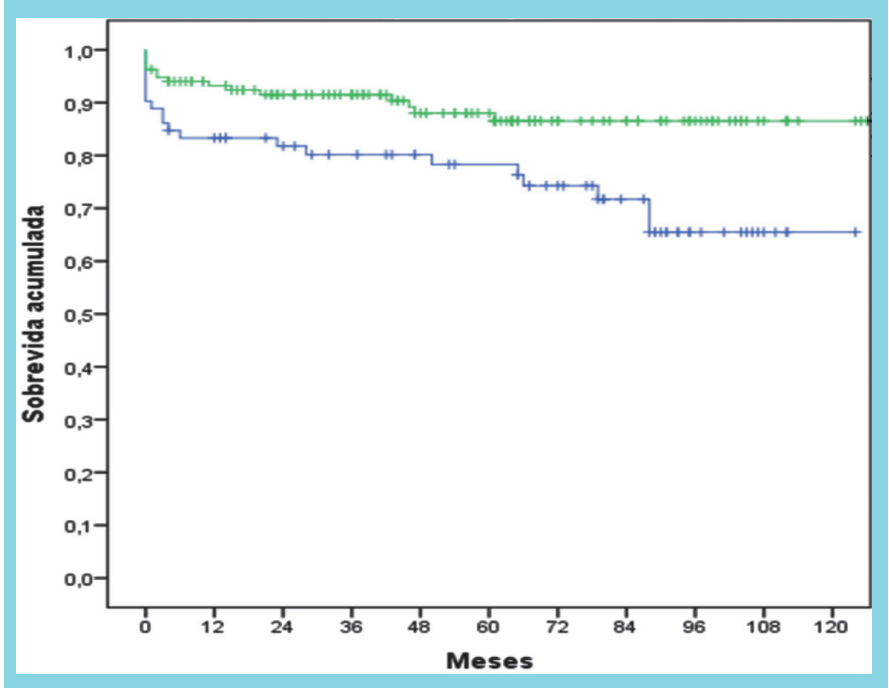


reumáticas y pudimos iniciar la cirugía de reparación de esta válvula. Se presentan los resultados de la serie inicial de pacientes intervenidos de reparación de válvula mitral durante estos años.

Debido a los múltiples mecanismos que pueden contribuir a la IM, nuestra cohorte es variada e incluye tanto a pacientes con IM primarias por enfermedad degenerativa y secuelas de endocarditis, e IM secundarias asociadas a enfermedad coronaria y a dilatación ventricular por patología aórtica.

En la reparación de la IM utilizamos principalmente las técnicas descritas por Carpentier (anillos, parches, resección cuadrangular, transposición de cuerdas). ${ }^{10,11}$ En algunos casos de prolapso del velo anterior utilizamos la transposición de cuerdas desde el velo posterior (en las válvulas con exceso de tejido) $)^{12}-14$, pero en la mayoría preferimos el implante de neocuerdas de PTFE descrito originalmente en forma experimental por Vetter y desarrollada posteriormente por otros autores. ${ }^{15-19}$ En el prolapso de velo posterior utilizamos resecciones cuadrangulares en las válvulas con exceso de tejido y resecciones triangulares cuando la cantidad de tejido del velo era normal o el anillo no estaba tan dilatado. ${ }^{20}$. Hubo un paciente con rotura de músculo papilar post infarto al que se reimplantó el músculo.

En la mayoría de los pacientes con IM secundaria a dilatación y disfunción ventricular ésta estaba asociada a insuficiencia aórtica. En esos casos realizamos la anuloplastía mitral como complemento al reemplazo valvular aórtico. En los casos de IM secundaria de causa isquémica realizamos una anuloplastía como complemento a la revascularización del corazón.

Todas las reparaciones incluyeron el implante de un anillo protésico. Esto no solo permite disminuir los diámetros del anillo mitral, sino también disminuir la tensión sobre la reparación, restablecer la forma de la válvula y aproximar velo anterior al posterior, aumentando la superficie de coaptación. ${ }^{4}$ A pesar del uso de diferentes modelos (rígidos, flexibles, completos, incompletos, etc.) los resultados de la reparación no difirieron entre los diferentes tipos de anillos, lo que es similar a lo descrito por otros autores. 21,22

Todas las reparaciones fueron evaluadas a la salida de circulación extracorpórea con ETE realizado por el anestesiólogo. ${ }^{23}$ En ellas se comprobó la ausencia IM residual, la superficie de coaptación (idealmente superior a $6 \mathrm{~mm})$, la ausencia de SAM y la ausencia de gradiente transvalvular. Estos parámetros se asocian a la durabilidad de la reparación en el largo plazo. ${ }^{24}$
El éxito de la reparación se logró en más del 95\% de los casos. Creemos que esto se debe a una buena selección de los pacientes en base al análisis preoperatorio de la válvula con ecocardiografía transtorácica y transesofágica. Este análisis permitió diagnosticar adecuadamente el mecanismo de la insuficiencia y con ello la realización de una reparación dirigida a corregir dicho mecanismo con los diferentes procedimientos quirúrgicos existentes. La mortalidad operatoria fue baja $(5,8 \%)$ y fue mayor en los pacientes con IM secundaria (9,7\%, versus 3,7\%). Esta mortalidad es más alta que la reportada por diferentes registros internacionales para la reparación mitral no asociada a cirugía coronaria, donde varía de 1 a $2 \%{ }^{25}$, 26. Sin embargo, se aproxima a la esperada según el perfil de riesgo de los pacientes calculado por EuroSCORE. En nuestro país la serie más grande de cirugías de reparación mitral fue publicada el 2014 por Zalaquett et al, quienes reportaron 322 pacientes intervenidos por diferentes causas. En esa serie la mortalidad operatoria global fue $7,5 \% ; 13 \%$ para los pacientes con IM isquémica y 4,4\% para la IM no isquémica ${ }^{27}$. En esta última se incluye la enfermedad reumática, endocarditis, cirugía bivalvular y cirugía de urgencia. En pacientes con insuficiencia mitral degenerativa aislada operados en forma electiva la mortalidad fue $0,7 \%$.

En la serie presentada los pacientes con IM secundaria tenían un riesgo operatorio mayor, un diámetro y disfunción ventricular más significativas, más enfermedad coronaria y/o aórtica asociadas y se realizaron más cirugías combinadas con tiempos de circulación extracorpórea más largos que aquellos con IM primaria. Sin embargo, la diferencia de mortalidad operatoria entre grupos no alcanzó significación estadística, lo que puede atribuirse a un relativamente bajo número de pacientes incluidos.

Los resultados publicados para la reparación valvular mitral en el largo plazo son muy buenos, con sobrevida global y libre de re intervención superiores a 90\% a 10 años. Esto varía según factores como la capacidad funcional preoperatoria o el velo mitral comprometido. ${ }^{28,29}$. En nuestra serie la sobrevida global a 5 años fue de $83,5 \%$, $90 \%$ para las primarias y $78 \%$ para las secundarias, en concordancia con los mismos factores ya comentados 30 . De los pacientes que fallecieron, solo el $50 \%$ tuvo relación con la patología cardiovascular. La tasa de reintervenciones en el seguimiento fue muy baja, aunque debemos esperar un tiempo mayor (10 años) para ver si esta tendencia se mantiene. 


\section{Limitaciones}

Se trata de una serie de pacientes seleccionados, operados en un periodo de 11 años en un solo centro. Los resultados están influenciados por el sesgo en la selección y la experiencia del equipo quirúrgico. Debido a que se trata de un centro de referencia, algunos pacientes provenientes de otros hospitales pudieron requerir de una reintervención que no fue realizada en el centro. El seguimiento de 5 años es corto y se debe esperar un tiempo mayor para ver si estos resultados se mantienen en el tiempo.

\section{Conclusiones:}

La cirugía de reparación de válvula mitral es el tratamiento de elección para pacientes con insuficiencia mitral, tanto primaria como secundaria. La evaluación del mecanismo de la insuficiencia por ecocardiografía y el uso de diferentes técnicas de reparación permitió conservar la válvula nativa en el $95 \%$ de los pacientes con una baja mortalidad operatoria, excelente sobrevida y ausencia de reintervenciones en el seguimiento.

\section{Referencias}

1. LUNG B, VAHANIAN A. Epidemiology of Acquired Valvular Heart Disease Canadian Journal of Cardiology 2014; 30, Issue 9: $962-970$.

2. J JOUAN. Mitral valve repair over five decades. Ann Cardiothorac Surg 2015; 4(4): 322 - 334

3. CARPENTIER A, DELOCHE A, DAUPTAIN J, SOYER R, BLONDEAU P, PIWNICA A, et al. A new reconstructive operation for correction of mitral and tricuspid insufficiency. $\mathrm{J}$ Thorac Cardiovasc Surg 1971; 61: 1-13.

4. CARPENTIER A. Cardiac valve surgery-The "French correction”. J Thorac Cardiovasc Surg 1983; 86: 323-37.

5. SAVAGE EB, FERGUSON TB, DISESA VJ. Use of mitral valve repair: analysis of contemporary United States experience reported to the Society of Thoracic Surgeons National Cardiac Database. Ann Thorac Surg 2003; 75: 820-5.

6. GAMMIE JS, SHENG S, GRIFFITH BP, PETERSON ED, RANKIN JS, O'BRIEN SM, et al. Trends in mitral valve surgery in the United States: results from the Society of Thoracic Surgeons Adult Cardiac Surgery Database. Ann Thorac Surg 2009; 87:1431 -7.

7. DUBERNET J, MORÁN S, MATURANA G, URZÚA J, FAJURI A. Cirugía reparadora como alternativa al reemplazo valvular. Rev Chil Cir 1977; 29: 677 - 9.
8. ZALAQUETT R. Cirugía reparadora de la insuficiencia valvular mitral. Rev Chil Cir 1994; 46: 127-36.

9. EL SABBAGH A, REDDY Y, NISHIMURA RA. Mitral valve regurgitation in the contemporary era: Insights into diagnosis, management, and future directions. J Am Coll Cardiol Img 2018; 11: $628-43$.

10. CARPENTIER A, CHAUVAUd S, FABIANI JN, et al. Reconstructive surgery of mitral valve incompetence. Ten year appraisal. J Thorac Cardiovasc Surg 1980; 79: 338-48.

11. DELOCHE A, JEBARA VA, RELLAND JYM, et al. Valve repair with Carpentier techniques. The second decade. J Thorac Cardiovasc Surg 1990; 99: 990-1002.

12. SOUSA UVA M, GRARE P, JEBARA V, FUZELIER JF, PORTOGHESE M, ACAR C, et al. Transposition of chordae in mitral valve repair. Mid-term results. Circulation 1993: 88: II35-38.

13. SALATI M, SCROFANI R, FUNDARO P, CIALFI A, SANTOLI C. Correction of anterior mitral prolapse. Results of chordal transposition. J Thorac Cardiovasc Surg 1992; 104: $1268-1273$.

14. SALATI M, MORIGGIA S, SCROFANI R, SANTOLI C. Chordal transposition for anterior mitral prolapse: early and long-term results. Eur J Cardiothorac Surg 1997; 11: 268-273. 
15. VETTER HO, BURACK JH, FACTOR SM, FRATER RWM. Replacement of chordae tendineae of the mitral valve using the new expanded PTFE suture in sheep. in: Bodnar E. Yacoub M. Biologic and Bioprosthetic Valves. Yorke Medical Books, New York1986: 772-784.

16. DAVID TE. Replacement of chordae tendineae with expanded polytetrafluoroethylene sutures. J Cardiac Surg. 1989; 4: 286-290.

17. ZUSSA C, FRATER RWM, POLESEL E, GALLONI M, VALFRÈ C. Artificial mitral valve chordae: experimental and clinical experience. Ann Thorac Surg. 1990; 50: 367-373.

18. DAVID TE, OMRAN A, ARMSTRONG S, SUN Z, IVANOV J. Long-term results of mitral valve repair for myxomatous disease with and without chordal replacement with expanded polytetrafluoroethylene sutures. J Thorac Cardiovasc Surg 1998; 115: 1279-1286.

19. VON OPPELL UO, MOHR FW. Chordal replacement for both minimally invasive and conventional mitral valve surgery using premeasured gore-tex loops. Ann Thorac Surg 2000; 70: 2166-2168.

20. DA COL U, DI BELLA I, BARDELLI G, KOUKOULIS G, RAMONI E, RAGNI T. Triangular resection and folding of posterior leaflet for mitral valve repair. J Card Surg. 2006; 21(3):274-6.

21. RUBENSTEIN F, REICHART B, LETSOU GV. Alternatives in selection of rings for mitral annuloplasty. Curr Opin Cardiol 2001, 16:136-139.

22. CHEE T, HASTON R, TOGO A, RAJA SH. Is a flexible mitral annuloplasty ring superior to a semi-rigid or rigid ring in terms of improvement in symptoms and survival? Interact CardioVasc Thorac Surg 2008; 7: 477-484.
23. OMRAN AS, WOO A, DAVID TE, et al. Intraoperative transesophageal echocardiography accurately predicts mitral valve anatomy and suitability for repair. J Am Soc Echocardiogr 2002;15:950-7.

24. SAIKI Y, KASEGAWA H, KAWASE M, OSADA H, OOTAKI E. Intraoperative TEE during mitral valve repair: does it predict early and late postoperative mitral valve dysfunction? Ann Thorac Surg. 1998; 66(4): 1277-81.

25. THE EUROPEAN ASSOCIATION FOR CARDIO-THORACIC SURGERY. Fourth EACTS adult cardiac surgical database report 2010. Henley-on-Thames, UK Dendrite Clinical Systems Ltd; ISBN 9781-9039-682-60.

26. GAMMIE J, CHIKWE J, BADHWAR V, THIBAULT DP, VEMULAPALLI S, THOURANI V et al. . Isolated Mitral Valve Surgery: The Society of Thoracic Surgeons Adult Cardiac Surgery Database Analysis. Ann Thorac Surg 2018;106:716-27.

27. ZALAQUETT R, LATORRE G, ALMEIDA J, MENESES A, MUÑOZ C, CÓRDOVA S. Veinte años de cirugía reparadora de la válvula mitral. Rev Med Chile 2014; 142: 1089-1098.

28. BRAUNBERGER E, DELOCHE A, BERREBI A, ABDALLAH F, CELESTIN JA, MEIMOUN P, et al. Very LongTerm Results (More Than 20 Years) of Valve Repair With Carpentier's Techniques in Nonrheumatic Mitral Valve Insufficiency. Circulation. 2001;104[suppl I]: I-8-I-11.

29. TIRONE E. DAVID. Durability of mitral valve repair for mitral regurgitation due to degenerative mitral valve disease. Ann Cardiothorac Surg 2015; 4: 417-421.

30. GROSSI EA, BIZEKIS CS, LAPIETRA A, DERIVEAUX CC, GALLOWAY AS, RIBAKOVE GH, et al. Late results of isolated mitral annuloplasty for "functional" ischemic mitral insufficiency. J Card Surg 2001; 16: 328-32. 